\title{
A New Form of Fuzzy Hausdorff Space and Related Topics via Fuzzy Idealization
}

\author{
A. A. Salama \\ Dept. of Maths and Computer Scinece, Faculty of Scineces, Port Said University, Egypt.
}

\begin{abstract}
In this paper, fuzzy L-open sets due to Abd El-Monsef et al. [4] are used to introduce a new separation axiom and new type of function in fuzzy topological ideals spaces. Some the basic properties of fuzzy L-irresolute functions, as well as the connections between them, are investigated. Possible application to superstrings and $\zeta^{\infty}$ space-time are touched upon.
\end{abstract}

\section{Introduction.}

The concepts of fuzzy sets and fuzzy set operations were first introduced by Zadeh[11]. Subsequently, Chang [6] defined the notion of fuzzy topology. Since then various aspects of general topology were investigated and carried out in fuzzy sense by several authors of this field. The local properties of a fuzzy topological space, which may also be in cretin cases the properties of the whole space, are important field for study in fuzzy topology by introducing the notion of fuzzy ideal and fuzzy local function [7,10 ]. In 2001, Abd El-Monsef et al. [4] defined and studied the notion of fuzzy L-open set in fuzzy topological space. In the present paper, we introduce and characterize the notion of fuzzy L-Hausdorff spaces which is a generlization of some fuzzy concepts by using a fuzzy L-open sets, we also define the class of fuzzy irresolute functions via fuzzy topological ideals and investigate its relation to fuzzy L-Hausdorff spaces and some topological consepts.

\section{Terminologies.}

Throughout this paper, by $(\mathrm{X}, \tau)$ we mean a fts in the sense of Chang's [ 6 ]. A fuzzy point in $\mathrm{X}$ with support $x \in X$ and value $\varepsilon(0<\varepsilon \leq 1)$ is denoted by $x_{\varepsilon}$.A fuzzy point $\mathrm{x}_{\varepsilon}$ is said to be contained in a fuzzy set

$\mu$ in X iff $\varepsilon \leq \mu(x)$ and this will denoted by $X_{\varepsilon} \in \mu$ [8]. For a fuzzy set $\mu$ in X, $c l(\mu), \mu^{\mathrm{c}}$ and $\operatorname{int}(\mu)$ will respectively denote closure, complement and interior of $\mu$. The constant fuzzy sets taking values 0 and 1 on $\mathrm{X}$ are denoted by $0_{x}, 1_{x}$, respectively. A fuzzy set $\mu$ is said to be quasi- coincident with a fuzzy set $\eta$, denoted by $\mu$ $\mathrm{q} \eta$, if there exists $x \in \mathrm{X}$ such that $\mu(\mathrm{x})+\eta(\mathrm{x})>1[8]$. Obviously, for any two fuzzy set $\mu$ and $\eta$, $\mu \mathrm{q} \eta$ will simply $\eta q \mu$. A fuzzy set $\rho$ in a fts $(X, \tau)$ is called a q-nbd of a fuzzy point $x_{\varepsilon}$ iff there exists a fuzzy open set $v$ such that $\mathrm{x}_{\varepsilon} \mathrm{q} v \subseteq \rho[6,8]$. We will denote the set of all q-nbd of $\mathrm{x}_{\varepsilon}$ in $(X, \tau)$ by $N\left(\mathrm{x}_{\varepsilon}\right)$. A fts $(X, \tau)$ is said to be a fuzzy extremely disconnected [1] (F.E.D in short) if the closure of every fuzzy open set in $\mathrm{X}$ is fuzzy open set. A fuzzy set $\mu$ for a fts $(X, \tau)$ is called fuzzy. $\alpha$-open [1] (resp, $\beta$-open [1], preopen [ 6 ] ) iff $\mu \leq \operatorname{int}(\operatorname{cl}(\operatorname{int}(\mu))$ ) (resp. $\mu \leq \operatorname{cl}(\operatorname{int}(\operatorname{cl}(\mu))), \mu \leq \operatorname{int}(\operatorname{cl}(\mu)))$. A non-empty collection of fuzzy sets $\mathrm{L}$ of a set $\mathrm{X}$ is called a fuzzy ideal [ 7,9] iff (i) $\mu \in \mathrm{L}$ and $\eta \subseteq \mu \Rightarrow \eta \in \mathrm{L}$ (heredity), (ii) $\mu \in \mathrm{L}$ and $\eta \in \mathrm{L} \Rightarrow \mu \cup \eta \in \mathrm{L}$ (finite additivity) Fuzzy closure operator of fuzzy set $\mu$ (in short $\operatorname{cl}^{*}(\mu)$ ) is define $\operatorname{cl}^{*}(\mu)=\mu \vee \mu^{*}$, and $\tau^{*}(\mathrm{~L})$ be the fuzzy topology generated by $\mathrm{cl}^{*}$ i.e. $\tau^{*}(\mathrm{~L})=\left\{\mu\right.$ : $\left.\mathrm{cl}^{*}(\mu)^{\mathrm{c}}=\mu^{\mathrm{c}}\right\}$ [10] .The fuzzy local function [10] $\mu^{*}(\mathrm{~L}, \tau)$ of a fuzzy set $\mu$ is the union of all fuzzy points $\mathrm{x}_{\varepsilon}$ such that if $\rho \in \mathrm{N}\left(\mathrm{x}_{\varepsilon}\right)$ and $\lambda \in \mathrm{L}$ then there is at least one $\mathrm{r} \in \mathrm{X}$ for which $\rho(\mathrm{r})+\mu(\mathrm{r})$ $-1>\lambda(\mathrm{r})$. The fuzzy ideal of fuzzy nowhere dense sets is $L_{n}=\left\{\mu \in I^{X}: \operatorname{int}(\operatorname{cl}(\mu))=O_{X}\right\}$. [ 2,3 ].

Definitioin2.1.Given $(X, \tau)$ be a fts with fuzzy ideal $\mathrm{L}$ on $\mathrm{X}, \mu \in I^{X}$ and $\mu$ is called

i. Fuzzy *-dense in itself if $\mu \leq \mu^{*}$ [2].

ii. Fuzzy scattered if contains no non empty fuzzy dense -in -itself [4] .

iii. Fuzzy L-open iff $\mu \leq \operatorname{int}\left(\left(\mu^{*}\right)\right)$ [4].

iv. Fuzzy L-closed set if its complement is fuzzy L-open set [ 4].

We will denote the family of all fuzzy L-open (resp.L-closed)by FLO(X)(resp.FLC(X)). 
Definitioin2.2. [2]. A fts $(X, \tau)$ is said to be fuzzy resolvble space if there is a fuzzy dense subset $\mu \in I^{X}$ for wich $\mu^{c}$ is also fuzzy dense equivalently, it can be expressed as the disjoint union of two fuzzy dense subsets.

Lemma 2.1.[2]. A fts $(X, \tau)$ is said to be fuzzy maximally irrresolvble space if it is fuzzy dense in-itself and has the property that every fuzzy dense subset of $(X, \tau)$ is fuzzy open.

Recall that fuzzy topological spaces having the property that their fuzzy dense subsets are fuzzy open are called fuzzy submaximal .

Definitioin2.3 . A fts $(X, \tau)$ is said to be fuzzy $\beta$-Hausdorff if for every two different fuzzy points $p, q$ of $X$, there exist disjoint fuzzy $\beta$-open sets $\mu$ and $\rho$ of $X$ such that $p \in \mu$ and $q \in \rho$.

Definitioin2.3 [4 ].A function $f:(X, \tau) \rightarrow(Y, \sigma)$ with a fuzzy ideal $\mathrm{L}$ on $\mathrm{X}$ is said to be fuzzy Lcontinuous if for every $\mu \in \sigma, f^{-1}(\mu) \in F L O(X)$.

Definition 2.2 [ 4].For a fts $(X, \tau)$ with fuzzy ideal $\mathrm{L}$ on $\mathrm{X}$, said to be compatible with $\mathrm{L}$, denoted by $\tau \sim L$, if for every fuzzy set $v$ of $\mathrm{X}$, and for all fuzzy point $x_{\varepsilon} \in v$, there exists a q-nbd $\mu$ of $x_{\varepsilon}$ such that $v(r)+\mu(r)-1 \leq \rho(r)$ hold for every $r \in X$ and for some $\rho \in L \quad ;$ then $v \in L$.

\section{Fuzzy L-Hausdorff Space}

Definition 3.1. A fts $(X, \tau)$ with fuzzy ideals $\mathrm{L}$ is called fuzzy L-Hausdorff space if for every pair of fuzzy singletons $p, q$ in $X$ with different supports, there exists $\mu, \rho \in F L O(X)$ with $p \in \mu, q \in \rho$ and $\mu \wedge \rho=O_{X}$.

Then we say that the points $p$ and $\mathrm{q}$ are fuzzy separated.

The following theorem gives an equivalent definition for a fuzzy

L-Hausdorff Space .

Theorem 3.1.A fts $(X, \tau)$ with fuzzy ideals $\mathrm{L}$ be L-Hausdorff iff if for each pair of fuzzy singletons $p, q$ in $X$ with different supports, there exists $\mu_{p} \in F L O(X)$ such that $p \in \mu_{p} \leq \operatorname{cl}\left(\mu_{p}\right) \leq\{q\}^{c}$.

Proof Let $(X, \tau)$ with fuzzy ideals $\mathrm{L}$ is fuzzy L-Hausdorff space and fuzzy singletons $p, q$ with different supports in $X$. Then there exists a fuzzy L-open set $\mu$ such that $p \in \mu, q \notin \rho$ and a fuzzy L-open set $\rho$ such that $\mu \wedge \rho=O_{X}$. Then $p \in \mu_{p} \leq c l\left(\mu_{p}\right) \leq \rho^{c} \leq\{q\}^{c}$.Conversely, let $p, q$ be two fuzzy singletons fuzzy $p, q$ in $X$ with different supports in $X$ and $\mu \in F L O(X)$ such that $p \in \mu_{p} \leq c l\left(\mu_{p}\right) \leq\{q\}^{c}$. Then $q \in(\operatorname{cl}(\mu))^{c}=\rho$ which is fuzzy open, $\mu \wedge \rho=\mu \wedge(\operatorname{cl}(\mu))^{c}=O_{X}$. Therefore $(X, \tau)$ with fuzzy ideal $\mathrm{L}$ is fuzzy $\mathrm{L}-$ Hausdorff.

Theorem 3.2. Every fuzzy L-Hausdorff space is fuzzy $\beta$ - Hausdorff .

Proof . Let $(X, \tau)$ be a fts with fuzzy ideal L is a fuzzy L-Hausdorff space. For every fuzzy L-open subset $\mu$ we have $\mu \leq \mu^{* \circ} \leq \operatorname{cl}\left(\mu^{* \circ}\right)$.It is clear that $\mu^{*} \leq \operatorname{cl}(\mu)$, since $o_{x} \in L$; hence $\mu \leq \operatorname{cl}\left(\operatorname{cl} \mu^{\circ}\right)$ and thus $(X, \tau)$ is fuzzy $\beta$ - Hausdorff .

The following example shows that the reverse of Theorem 3.2 is not generally true .

Example 3.1. Let $X=\{(x, 1),(y, 1)\}, \tau$ be the fuzzy discrete topology on $X$ and $L=\left\{I^{X}\right\}$. Thus $X$ is fuzzy Hausdorff and consequently fuzzy $\beta$ - Hausdorff, but not fuzzy L-Hausdorff, Since $F L O(X)=O_{X}$. Theorem 3.3. For a fts $(X, \tau)$ with fuzzy ideal $\mathrm{L}$ we have

i. If $(X, \tau)$ is a fuzzy Hausdorff and every fuzzy open subspace is fuzzy *-dense in itself, then $(X, \tau)$ with $L$ is a fuzzy L-Hausdorff. 
ii. If $(X, \tau)$ is fuzzy Hausdorff and $\mathrm{L}$ is $\tau$ - boundary, then $(X, \tau)$ with fuzzy ideal $\mathrm{L}$ is a fuzzy LHausdorff.

Proof

i. Obvious, since for every fuzzy open set $\mu \in I^{X}$ we have $\mu=$ int $\mu \leq$ int $\mu^{*} \leq \operatorname{cl}\left(\mu^{* \circ}\right)$.For note that $\mu \leq \mu^{*}$ since $\mu$ is fuzzy *-dense- in-itself .

ii. Clear from the definition and the fact that $\mu \leq \mu^{*}$ Note that if $L, J$ are two fuzzy ideal on $\mathrm{X}$ and $L \leq J$, then $\mu^{*}(J) \leq \mu^{*}(L)$ and the following result holds

Theorem 3.4.If if $L, J$ are two fuzzy ideal on $X$ and $L \leq J$, then $F L O(X, \tau, J) \leq F J O(X, \tau, L)$, so that $(X, \tau)$ with fuzzy ideal L is fuzzy $J-$ Hausdorff.

Proof .Obvious

Theorem 3.5 . Let $(X, \tau)$ be a fts with fuzzy ideal $\mathrm{L}$ is a fuzzy L-Hausdorff space and $\mu \in I^{X}$.Then

i. $\quad$ If $\mu$ is fuzzy open, then $\mu$ is fuzzy L- Hausdorff space .

ii. If $\mu$ is fuzzy is $\alpha$-open, then $\mu$ is fuzzy $\beta$-Hausdorff.

Proof .

i. Follows directly from Lemma2.1 [3] and the fact that for a fts $(X, \tau)$ with fuzzy ideal L, if $\rho \in \tau$ and $\mu$ be a subspace of $(X, \tau)$ then $\rho \wedge(\rho \wedge \mu)^{*} \leq(\rho \wedge \mu)^{*}$.

ii. $\quad$ Since every L-open set is fuzzy $\beta$-open and the intersection of a fuzzy $\beta$-open and fuzzy $\alpha$-open set is fuzzy $\beta$-open in fuzzy $\alpha$-open set, the theorem is clear .

Thereom3.6.Let with fuzzy ideal be fts with the following property if are two fuzzy singletons with different supports, then there exist such fuzzy Hausdorff space Y and fuzzy L-contiuaus function that .Then is fuzzy

L-Hausdorff .

Proof . Obvious

\section{Fuzzy L- irresolute functions}

Definition 4.1. A function $f:(X, \tau) \rightarrow(Y, \sigma)$ with fuzzy ideal $\mathrm{L}$ on $\mathrm{X}$ is called fuzzy L- irresolute if $f^{-1}(\mu) \in F L O(X)($ resp.FLC $(X))$ for every $\mu \in F L O(Y)($ resp.FLC $(Y))$.

Theorem 4.1. if a function $f:(X, \tau) \rightarrow(Y, \sigma)$ with fuzzy ideal $\mathrm{L}$ on $\mathrm{X}$ is fuzzy L- irresolute then for any fuzzy L-open set $\mu \in \mathrm{I}^{Y}$ we have $c l\left(f^{-1}\left(\mu^{*}\right)\right) \leq f^{-1}\left(c l\left(\mu^{*}\right)\right)$.

Proof Since $\mu \leq \operatorname{int}\left(\mu^{*}\right) \leq \operatorname{cl}\left(\mu^{*}\right)$ this implies $c l\left(f^{-1}\left(\mu^{*}\right)\right) \leq f^{-1}\left(c l\left(\mu^{*}\right)\right)$. and since $f$ is fuzzy Lirresolute the theorem is proved .

Theorem 4.2. For a function $f:(X, \tau) \rightarrow(Y, \sigma)$ with fuzzy ideal $\mathrm{L}$ and $\mathrm{J}$ on $\mathrm{X}$ and $\mathrm{Y}$ respectively. The following the following are equivalent
i. $\quad f$ fuzzy L- irresolute ;
ii. the inverse image of each fuzzy L-closed in $(Y, \sigma)$ with fuzzy ideal $\mathrm{J}$ is fuzzy L-closed in $(X, \tau)$ with fuzzy ideal L;
iii. containing $f(p)$ such that $f(\mu) \leq \rho$.

Proof. The proof is obvious and thus omitted.

Theorem 4.3. Let $f$ be a one -to-one fuzzy L- irresolute map from fts $(X, \tau)$ with fuzzy ideal $\mathrm{L}$ into a fts $(Y, \sigma)$ with fuzzy ideal J . If $(Y, \sigma)$ is fuzzy L- Hausdorff, then $(X, \tau)$ with fuzzy ideal L is fuzzy LHausdorff .

Proof Let $p, q$ be a pair of fuzzy singletons in X with different supports. Then $f(p) \neq f(q)$ and moreover $f(p)$ and $f(q)$ are fuzzy L-separated in $(Y, \sigma)$ by fuzzy L-open sets $\mu$ and $\rho$, respectively . the disjoint fuzzy sets $f^{-1}(\mu)$ and $f^{-1}(\rho)$ are fuzzy L-open ,since $f$ is fuzzy L- irresolute and contain $p, q$, respectively. Then, we have that $(X, \tau)$ is fuzzy L- Hausdorff 
Theorem 4.4. Let $f:(X, \tau) \rightarrow(Y, \sigma)$ and $g:(Y, \sigma) \rightarrow(Z, \theta)$ be two functions, where $L, J$ and $K$ are fuzzy ideals on $\mathrm{X}, \mathrm{Y}$ and $\mathrm{Z}$, respectively.Then $g \circ f$ is fuzzy L-irresolute, if both $f$ and $\mathrm{g}$ are fuzzy L-irresolute.

Proof . obvious.

Theorem 4.5. Let $f:(X, \tau) \rightarrow(Y, \sigma)$ where $L, J$ are fuzzy ideals on $\mathrm{X}$ and $\mathrm{Y}$ respectively. Then

i. If $f$ is fuzzy L-irresolute and each open subspace of Y is fuzzy *-dense-in itself, then $f$ is fuzzy Lcontinuous .

ii. If $f$ is fuzzy L-continuous and $(Y, \sigma)$ is fuzzy sub- maximal E.D, then $f$ is fuzzy L- irresolute .

iii. If $L=J=\left\{O_{X}\right\}$, each fuzzy irresolute and $\beta$ - irresolute is equivalent .

iv. If $L=J=\left\{I^{X}\right\}$, fuzzy L- irresoluteness and fuzzy $\beta$-irresoluteness coincide.

Proof .

i. Clear, since each fuzzy open and fuzzy *-dense in-it-self is fuzzy L-open and consequently fuzzy L-open (see [4]).

ii. Clear, since each fuzzy L- open set is fuzzy $\beta$-open and thus fuzzy open from Lemma 2.1 [ 4 ] .

iii. , iv Follow from Lemma 2.1. [ 4 ] .

Theorem 4.6. if $f:(X, \tau) \rightarrow(Y, \sigma)$ with fuzzy ideal $\mathrm{L}$ is an injective fuzzy L-irresolute map then $(Y, \sigma)$ is fuzzy L-Hausdorff space and then $(X, \tau)$ is fuzzy L- Hausdorff space.

Proof Let $\rho_{1}, \rho_{1}$ be any two distinct fuzzy singletons in $\mathrm{X}$, thus we have $\rho_{1}=f^{-1}\left(q_{1}\right)$ and $\rho_{2}=f^{-1}\left(\mathrm{q}_{2}\right)$, where $q_{1}$ and $q_{2}$ are distinct fuzzy singletons in $(Y, \sigma)$.Since $(Y, \sigma)$ is fuzzy L-Hausdorff, then there exist $\mu, \rho \in F L O(Y)$ such that $q_{1} \in \mu, q_{2} \in \rho$ and $\mu \leq \mathrm{Y}-\rho$, thus $f^{-1}\left(q_{1}\right) \in f^{-1}(\mu), f^{-1}\left(q_{2}\right) \in f^{-1}(\mu)$ and $f^{-1}(\mu) \leq X-f^{-1}(\rho)$ which means that $\mu \wedge \rho=O_{X}$ this implies $(X, \tau)$ is fuzzy LHausdorff space .

\section{References}

[1] M.E. Abd El-Monsef and M.H. Ghanim, On semi open fuzzy sets, Delta , J. Sci.5 (1981) 30-40.

[2] M.E. Abd El-Monsef; A.A. Nasef and A. A. Salama, Extensions of Fuzzy Ideals, Bull.Cal.Maths.Soc., 92 , (3) ( 2000 ) 181 -188

[3] M.E. Abd EI-Monsef; A.A.Nasef and A.A.Salama, Some Fuzzy Topological operators via Fuzzy ideales Chaos, Solitons \& Fractals.(12)(13),(2001), 2509-2515.

[4] M.E. Abd El-Monsef; A.A. Nasef and A.A.Salama, Fuzzy L-open Sets and Fuzzy L-continuous Functions, Analele Universitatii din Timisoara .XL,fasc.Seria Matematica - Informatica(2),(2002),3-12

[5] K.K. Azad, On fuzzy semi continuity, fuzzy almost continuity and fuzzy weakly continuity, J. Math. Anal App. 82 (1981) 1 ĩ32.

[6] C.L. Chang, Fuzzy Topology Spaces, J. Moths. Anal - Apple 24 (1968) 128-189.

[7] R.A.Mahmoud, Fuzzy Ideals, fuzzy local functions and *-fuzzy topology, the Journal of Fuzzy Mathematics Vol.5, No.1, 1997 Los Angeles.

[8] Pu Pao - Ming and Liu Yang, Fuzzy Topology .1. Neighbour - hood structure of fuzzy point and Moore smith convergence, J. Math Anal. App. 76 (1980) 57ĩ-599.

[9] A.A.salama, Fuzzy Ideals on fuzzy topological spaces, Ph.D in Topology ,Tanta Univ., Egypt( 2002).

[10] D.Sarkar,Fuzzy ideal theory, Fuzzy local function and generated fuzzy topology, Fuzzy Sets and Systems 87 (1997) $117-123$

[11] L.A. Zadeh, Fuzzy Sets. In form and control 8(1965) 338-353. 